\section{ZEW}

Zentrum für Europäische Wirtschaftsforschung GmbH

Centre for European Economic Research

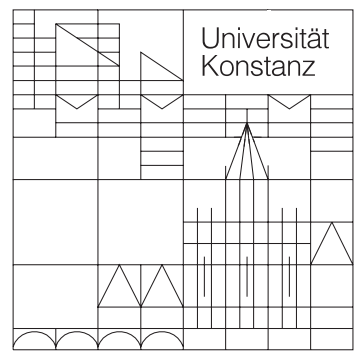

Rechts-, Wirtschafts- und Verwaltungswissenschaftliche Sektion Fachbereich

Wirtschaftswissenschaften

Diskussionspapiere der DFG-

Forschergruppe (Nr.: 3468269275):

Heterogene Arbeit: Positive und Normative Aspekte der Qualifikationsstruktur der Arbeit

Dominique Demougin,

Oliver Fabel

Autonomie der Universität und Professionalisierung ihrer Leitungsstrukturen

Mai 2005 
Diskussionspapier der Forschergruppe (Nr.: 3468269275) "Heterogene Arbeit: Positive und Normative Aspekte der Qualifikationsstruktur der Arbeit"

Nr. 05/05, Mai 2005

\section{Autonomie der Universität und Professionalisierung ihrer Leitungsstrukturen}

\section{Dominique Demougin}

School of Business and Economics

Humboldt University of Berlin

Spandauer Str. 1

10178 Berlin

mail: :demougin@wiwi.hu-berlin.de

phone: $\quad+49-30-2093-1341$

fax: $\quad+49-30-2093-1343$

\section{Oliver Fabel}

\author{
Fach D 144 \\ Universität Konstanz \\ 78457 Konstanz \\ Germany \\ mail : oliver.fabel@uni-konstanz.de \\ phone : +49-7531-88-2990 /-2992 \\ fax : $+49-7531-88-4456$
}

\section{Zusammenfassung:}

Autonomie der Universität und Professionalisierung ihrer Leitungsstrukturen:

Im Zuge der Professionalisierung der Leitungsstrukturen erfolgt die Unterstellung der Universitätsverwaltung als Agenten der akademischen Universitätsleitung. Dies verändert die Möglichkeiten der Universität und des Ministeriums, in bilateralen Budgetverhandlungen Rentenanteile abschöpfen zu können. Einigungen mit verhandlungsstarken Universitäten betreffen nur noch einen Sockelbetrag. Darüber hinaus kann die wissenschaftliche Leistung abnehmen, während gleichzeitig unproduktive Aktivitäten unternommen werden. Im Ergebnis kann sich das Ministerium gezwungen fühlen, durch gesetzliche, die Autonomie der Universität weiter beschneidende Regelungen die Verhandlungsmacht der Universität zu beschränken.

JEL Klassifikation Schlüsselwörter : L30, M40, M21 : Organisationsautonomie, Organisation des Controlling, Prinzipal-Agenten Ansatz, Verhandlungsmodell

Download/Reference : http://www.wiwi.uni-konstanz.de/forschergruppewiwil 


\title{
Autonomie der Universität und Professionalisierung ihrer Leitungsstrukturen
}

\author{
Oliver Fabel \\ Fachbereich Wirtschaftswissenschaften \\ Universität Konstanz* \\ Dominique Demougin \\ Wirtschaftswissenschaftliche Fakultät \\ Humboldt Universität zu Berlin ${ }^{\dagger}$
}

Manuskript des Vortrags zum Generalthema „Innovation und Institution", Pfingsttagung des Verbandes der Hochschullehrer für Betriebswirtschaftslehre, Kiel, 21. Mai 2005

*Prof. Dr. Oliver Fabel, Lehrstuhl für Betriebswirtschaftslehre, insbesondere Unternehmenspolitik, Fachbereich Wirtschaftswissenschaften, Sektion Recht, Wirtschaft und Verwaltung, Universität Konstanz, Fach D144, 78457 Konstanz; Tel.: +49-(0)7531-882990/-2992; Fax: +49-(0)7531-88-4456; E-Mail: oliver.fabel@uni-konstanz.de.

$\dagger$ Prof. Dominique Demougin, PhD, Walter Rathenau Lehrstuhl für Organisationstheorie, Wirtschaftswissenschaftliche Fakultät, Humboldt Universität zu Berlin, Spandauer Str. 1, 10178 Berlin; Tel.: +49-(0)30-2093-1341; Fax: +49-(0)30-2093-1343; E-Mail: demougin@wiwi.hu-berlin.de. 


\begin{abstract}
Autonomy of the University and the Professionalisation of its Organisation of Control:

Professionalizing the system of control in a still public system implies that the university administration becomes an agent of the academic board of the university rather than serving the interests of the state as the principal. This induced organizational change alters the bargaining positions of the ministery and the university which both aim to maximize their rent share in the budget negotiations. In particular, bargaining with powerful universities then only concerns a fixed transfer. Moreover, academic effort may decrease and there will be unproductive activities. As a result the ministery may feel pressed to restrain the bargaining power of the university by law that reduces the degree of university autonomy in strategic and organizational decision making.
\end{abstract}

Keywords: Organizational Autonomy, Organization of Control System, Principal Agent Approach, Bargaining Model

JEL-Classification: L30, M40, M21 


\section{Zusammenfassung}

\section{Autonomie der Universität und Professionalisierung ihrer Leitungsstruk-}

turen:

Im Zuge der Professionalisierung der Leitungsstrukturen erfolgt die Unterstellung der Universitätsverwaltung als Agenten der akademischen Universitätsleitung. Dies verändert die Möglichkeiten der Universität und des Ministeriums, in bilateralen Budgetverhandlungen Rentenanteile abschöpfen zu können. Einigungen mit verhandlungsstarken Universitäten betreffen nur noch einen Sockelbetrag. Darüber hinaus kann die wissenschaftliche Leistung abnehmen, während gleichzeitig unproduktive Aktivitäten unternommen werden. Im Ergebnis kann sich das Ministerium gezwungen fühlen, durch gesetzliche, die Autonomie der Universität weiter beschneidende Regelungen die Verhandlungsmacht der Universität zu beschränken.

Schlüsselworte: Organisationsautonomie, Organisation des Controlling, PrinzipalAgenten Ansatz, Verhandlungsmodell

JEL-Klassifikation: L30, M40, M21 


\section{Vorbemerkungen}

Der Arbeitstitel, der mit der Einladung zum Vortrag im Rahmen des Generalthemas „Innovation und Institutionen” während der Pfingsttagung des Verbandes der Hochschullehrer 2005 in Kiel verbunden war, lautete „Was sich an Universitäten geändert hat und was sich noch ändern wird". Dies ist sicher ein treffliches Thema zur Bearbeitung durch zwei Organisationsökonomen, die sich in der Vergangenheit mit der Ausgestaltung effizienter Anreiz-, Informations-, und Organisationssysteme befasst haben - mit allen Teilaspekten dessen also, was gemeinhin mit der Einführung „professioneller” Leitungs- und Controllingstrukturen in Universitäten verbunden wird. Allerdings ist es auch ein sehr weit gestecktes Thema für einen Überblicksbeitrag. Darüber hinaus scheint uns die Unzufriedenheit beider an dem Reformprozess beteiligten Parteien, der Universitäten und der Wissenschaftsministerien, weit über die Kritik einzelner Verfahren und Methoden des neuen Hochschulmanagements hinaus zu gehen.

Die Ministerien zeigen sich häufig unzufrieden mit der Leistung der Universitäten, drängen auf schnellere Umsetzung der internen Reformen zur Steigerung der Effizienz und Herstellung von Wettbewerbsfähigkeit. Durch gesetzliche Vorgaben werden daher neue Leitungs- und Organisationsstrukturen zwangsweise implementiert. Die Universitäten empfinden dies als Beschneidung ihrer Autonomie, deren (Wieder-)Herstellung ja von der Politik versprochenes Ziel des Reformprozesses ist. Zudem sehen sie sich durch die Zuweisung neuer Aufgaben zusätzlich belastet, da die entstehenden Kosten im Rahmen der inzwischen eingeführten Systeme leistungsbezogener Mittelvergaben kompensiert werden. Diese Systeme, die zwischen Universitäten und Ministerien verhandelt wurden und auch nachverhandelt werden, sowie die Steuerung über Zielvereinbarungen, die zwischen Ministerien und einzelnen Universitäten auszuhandeln sind, werden selbst dagegen als notwendiger Bestandteil eines finanzautonomen Universitätswesens angesehen.

Regelmäßig in ihrer Wirkung als „marktähnlich” beschrieben, stellen sie tatsächlich nur Wettbewerb zwischen den Universitäten als Agenten eines weiterhin als Prinzipal auftretenden Ministeriums her. Im Zuge der Professionalisierung der Leitungsstrukturen erfolgt nun eine tiefgreifende Veränderung dieses Prinzipal-Agenten Problems. Die Universitätsverwaltung wird als Agent der akademischen Uni- 
versitätsleitung unterstellt und nicht mehr als Teil der Landesverwaltung geführt. Dies verändert die Möglichkeiten der Universität und des Ministeriums, in bilateralen Budgetverhandlungen Rentenanteile abzuschöpfen. Einigungen mit verhandlungsstarken Universitäten betreffen dann nur noch einen Sockelbetrag. Bei unveränderter Verhandlungsmacht der beiden Akteure kann es zudem zu einer Einbuße an wissenschaftlicher Leistung kommen. Außerdem werden unproduktive Aktivitäten durchgeführt. Im Ergebnis kann sich das Ministerium also gezwungen fühlen, durch gesetzliche, die Autonomie der Universität weiter beschneidende Regelungen die Verhandlungsmacht der Universität zu beschränken. Dies reduziert aber die wissenschaftliche Leistungserstellung nur noch weiter.

Dieses positive Ergebnis unserer Analyse, die wir, nach einer kurzen Darstellung des gegenwärtigen Zustands allgemeiner Unzufriedenheit der Akteure im nächsten Abschnitt, im Abschnitt 3 auch formal entwickeln, zeigt vor allem, dass es nicht ausreicht, Veränderungen von Teilsystemen des Universitätswesens isoliert zu untersuchen, um beurteilen zu können, ob ein gestecktes Reformziel tatsächlich erreicht wird. Bereits die Wirkungsanalyse, die nur zwei Teilaspekte gegenwärtiger Reformanstrengungen, die Einführung von Budgetverhandlungen mit Ergebnisprüfung auf der Basis verifizierbarer Indikatorensysteme und die Professionalisierung der Universitätsverwaltung zusammenführt, deckt neue, bisher nicht beachtete Effekte auf. Bezogen auf die konkrete Fragestellung kommen wir dann im Abschnitt 4 zu dem Schluss, dass nur die weitgehende Auflösung des bestehenden PrinzipalAgenten Verhältnisses zwischen Staat und Universität die beschriebenen Probleme lösen kann.

\section{Autonomie der Universität: ein Zustandsbericht}

\subsection{Der Zusammenhang zwischen Autonomie und Profes- sionalisierung}

Art. 5 Abs. 3 GG schützt die Freiheit von Wissenschaft, Forschung und Lehre. Darüber hinaus begründet er „,die objektive Pflicht des Staates [...] funktionsfähige 
Institutionen für einen freien Wissenschaftsbetrieb zur Verfügung zu stellen". ${ }^{1}$ Dies impliziert gleichsam das Recht des Staates durch Gesetz und Verordnung diesen Wissenschaftsbetrieb zu organisieren. ${ }^{2}$ Insbesondere kann der Staat grundsätzlich den Grad der Dezentralisierung in diesem System festlegen. ${ }^{3}$ Erst die Erkenntnis, dass die zentrale Führung der Universitäten zu Bürokratie und erheblichen „X-Ineffizienzen” geführt hat, und nicht etwa ein unmittelbarer, grundgesetzlich verankerter Anspruch lässt also in jüngerer Zeit die (Wieder-)Herstellung der Universitätsautonomie zur zentralen Forderung in Ansätzen zur Reform des Universitätssystems werden.

Universitätsautonomie umfasst dabei regelmäßig die Rechts-, Finanz- und Organisationsautonomie. Insbesondere dem Recht, selbstständig und -verantwortlich über die eigene Organisation zu entscheiden, wird häufig eine besondere Rolle zugesprochen. So ist für Schiedermaier (1997, S. 15) klar, dass „,[Autonomie] ein Grundprinzip der modernen Bildungsverfassung [ist], die Freiheit von Forschung, Lehre und Studium gewährleistet. Dieser verfassungsrechtlichen Vorgabe hat die Universität [...] auch und vor allem in ihrer Organisation zu dienen." Ganz ähnlich versteht Müller-Böling Autonomie (2000, S. 45) als „Freiheit für eigenverantwortliches Handeln", die Organisationsautonomie - also "[d]ie Freiheit [...] sich zu organisieren, wie es ihren jeweiligen Bedürfnissen und ihrem generellen Leistungsauftrag entspricht und wie es Unterschiede in Größe, Tradition, Kultur usw. erfordern" voraussetzt.

Globalbudgets, die nach eigenen strukturellen Vorstellungen innerhalb der Universität auf Teileinheiten verteilt werden können, sind dann sicher eine Grundvoraussetzung für die Abkehr von staatlichen Strukturvorgaben. D. h., Organistionsautonomie ist nur durch finanzautonome Universitäten zu erreichen, die nicht mehr dem starren öffentlichen Haushaltsrecht unterworfen sind. ${ }^{4}$ Allerdings ist damit nun gerade das Interesse des Staates als Hauptfinancier im Kern betroffen, der sein bildungs- und forschungspolitisches Mandat wahrnehmen will und einer Demokratie auch wahrnehmen muss. ${ }^{5}$ Systeme leistungsbezogener Mittelvergabe und/oder Zielvereinbarungen gelten dann Vertretern der „Professionalisierungsrich-

\footnotetext{
${ }^{1}$ Geis (2003, S.244).

${ }^{2}$ Geis (2003) und Kirchhof (2003).

${ }^{3}$ Goerke (2003).

${ }^{4}$ Schock (2002).

${ }^{5}$ Vgl. wiederum Görke (2003).
} 
tung" in der hochschulpolitischen Debatte als adäquates Mittel zur Lösung von Interessenkonflikten. ${ }^{6}$

Genau an dieser Stelle unterscheiden sich aber die Einschätzungen der Effekte praktischer Reformvorhaben und -umsetzungen. Den einen gilt die Einführung von Instrumenten des „New Public Management” und u. U. weitergehender, der Privatwirtschaft entliehenen Managementmethoden als eine notwendige Voraussetzung zur Übernahme bisher staatlicher Aufgaben und damit zur Herstellung von Autonomie [Fedrowitz/Krasny/Ziegele (1999, S. 7)]. Die anderen sehen darin ein „Täuschungsmanöver" mit dessen Hilfe der Staat von der in der derzeitigen Lage eigentlich notwendigen Stärkung von „Kreativität statt Effizienz” in den Universitäten ablenken will [Schiedermaier (1997, S. 16)]. Behauptet wird, die „Ökonomisierung" der Universität gefährde gerade die Freiheit der Wissenschaft. ${ }^{7}$

Richtig ist aber zunächst nur, dass es sich hier um die Effekte von Zielvereinbarungen und leistungsbezogenen Mittelvergaben handelt, die grundsätzlich Verhandlungslösungen darstellen. Erstere werden zwischen der Gruppe der Landesuniversitäten und dem zuständigen Ministerium vereinbart, häufig nachverhandelt und immer mit Bestandsgarantien ausgestattet. Letztere sind auf Universitätsebene individualisiert. Da es sich hier um nicht-marktliche Mechanismen handelt, entsteht im Zuge der Umsetzung ein typisches „Agency”-Problem, das durch die Definition entsprechender Systeme von Performanzindikatoren beherrscht werden soll. ${ }^{8}$ Der oben beschriebene Disput über den Nutzen solcher Steuerungsmechanismen findet hier seinen Fortgang in der Diskussion darüber, ob der Erfolg der Leistungserstellung einer Universität überhaupt messbar ist oder, wenn dies zugestanden wird, wie adäquate Maße zu definieren sind..$^{9}$ Diese Auseinandersetzung soll jedoch an dieser Stelle ausdrücklich nicht nachvollzogen werden. ${ }^{10}$

Vielmehr ist offensichtlich, dass die Universität einmal einem solchen Finanzierungssystem unterworfen, ein entsprechendes Berichtswesen benötigt - und zwar

\footnotetext{
${ }^{6}$ Vgl. z. B. Fedrowitz/Krasny/Ziegler (1999) und Leszcensky/Orr (2004).

${ }^{7}$ Starbatty (2004).

${ }^{8}$ Vgl. wiederum Kirchhof (2003).

${ }^{9}$ Einen Überblick über die derzeit in Deutschland angewandten Indikatorensysteme geben wiederum Leszcensky/Orr (2004).

${ }^{10}$ Einer der Autoren hat sich damit bereits wiederholt auseinandergesetzt. Siehe hierzu Fabel/Heße (1999), Fabel/Hilgers (2001) oder Fabel/Lehmann/Warning (2002, 2003).
} 
sowohl ein externes, mit dessen Hilfe sich Erfolge im Sinne von Indikatorausprägungen ausweisen lassen, als auch ein internes, das der Universitätsleitung Informationen liefert, die geeignet sind, Strukturentscheidungen vorzubereiten und deren Auswirkungen zu überprüfen. Damit erfolgt zwangsläufig eine Abkehr vom Bild der traditionellen Universitätsverwaltung, deren Berichtspflicht ursprünglich auf die Weitergabe statistischer Daten beschränkt war, und deren Aufgabe im wesentlichen darin bestand, als Statthalter des Staates in der Universität auf die Einhaltung eines im Rechtssinne ordnungsgemäßen Betriebes hin zu wirken. Ein auf den Erfolgsausweis im Rahmen leistungsbezogener Mittelvergaben, auf den Nachweis der Erfüllung von Zielvereinbarungen sowie auf die Erstellung interner Steuerungsinformationen ausgerichtetes Berichtswesen impliziert aber eine Verwaltung, die als Agent ihrer Universität dient. ${ }^{11}$

Die Verwaltung wird also in einen Dienstleister umgewandelt, der Leistungen innerhalb der Universität erbringt. ${ }^{12}$ Es müssen darüber hinaus Leitungsstrukturen geschaffen werden, die in der Lage sind, Entscheidungen der Universitätsleitung innerhalb der Universität zu transportieren und durchzusetzen. So entsteht dann das Bild der „professionalisierten” Universität im Sinne der Entschließung der Hochschulrektorenkonferenz vom 8.6.2004. ${ }^{13}$ Obwohl sich dies immer noch deutlich von der Vorstellung abgrenzt, diese Entwicklung sei nur durch eine „Verberuflichung" des Leitungspersonals zu erreichen, ${ }^{14}$ folgt die Definition notwendiger Entscheidungs- und Aufsichtsgremien offensichtlich dem Vorbild der Wirtschaftspraxis.

Die damit verbundene Veränderung der Organisation des Universitätswesens ist daher tiefgreifend. Sie lässt aus einem Problem, in dem der Prinzipal „Ministerium” zwei Agenten, die akademische Universitätsleitung und die Universitätsverwaltung, unabhängig voneinander führt, ein Problem entstehen, in dem der zweite Agent dem ersten unterstellt wird, der allein gegenüber seinem Prinzipal auftritt. Aus informationsökonomischer Sicht tritt ein der Linie zugeordnetes an die Stelle eines

\footnotetext{
${ }^{11} \mathrm{Zu}$ den neuen Anforderungen an das Berichtswesen und seine Implementierung vgl. z. B. Leszcensky (2003).

${ }^{12}$ Vgl. z. B. das Selbstporträt der "Best Practise" Johannes Gutenberg Universität Mainz (2001).

${ }^{13}$ HRK (2004).

${ }^{14}$ Zur angelsächsischen Praxis vgl. hierzu Weiler (1998). Eine knappe kritisch-kontrastierende Diskussion findet sich z. B. in Heise (2002) und Hommelhoff (2002).
} 
zentralen Controlling. ${ }^{15}$

\subsection{Staatseingriffe und ,gefühlte" Autonomie}

Die Kritik am laufenden Reformprozess entzündet sich dennoch häufig allein an der Wirkung, die von Systemen der Performanzmessung ausgeht, und den weiteren Belastungen, die der Staat durch Auslagerung von Aufgaben den Universitäten auferlegt. Sie werden als Eingriffe eines letztlich nicht an einer Stärkung der Entscheidungsautonomie der Universität interessierten Staates gesehen. Unterstützung erhält diese Kritik dabei vor allem durch Erfahrungen, die an den Universitäten seit einigen Jahren gesammelt werden. So sind zwar 1998 im Zuge der Novellierung des Hochschulrahmengesetzes die Vorschriften zu Organisations- und Leitungsstrukturen entfallen, die so entstandene Regulierungslücke scheint jedoch weitgehend nur durch entsprechende und in Teilen sogar weiter gehende Regelungen auf Länderebene geschlossen worden zu sein.

Der Stifterverband für die deutsche Wirtschaft (2002) gelangt zu der Einschätzung, dass, bei durchaus existierenden länderspezifischen Unterschieden,

- die Rechtsform der Universität überwiegend entweder als staatliche Einrichtung oder öffentliche Körperschaft festgelegt ist und keine Übertragung bisher staatlich wahrgenommener Entscheidungsrechte auf Hochschulräte statt findet,

- die Organisationsautonomie mit wenigen Ausnahmen durch zu dichte Regulierung beschnitten und insbesondere die Binnengliederung der Universität in allen Ländern genehmigungspflichtig ist,

- in allen Ländern verbindliche Stellenpläne im Staatshaushalt vorgegeben werden,

- die Landesregierungen mit nur zwei Ausnahmen durch Genehmigungspflicht weiterhin mitentscheidend Einfluss auf die Einrichtung von Studiengängen nehmen.

\footnotetext{
${ }^{15}$ Chwolka (1996) analysiert die Entscheidung einer Unternehmensleitung zwischen zentralem und delegiertem Controlling bereits im Rahmen eines klassischen Prinzipal-Agenten Modells mit riskikoaversen Agenten und gewinnmaximierenden Prinzipal.
} 
Als - im Sinne einer Stärkung der Entscheidungsautonomie der Universität - bereits weit entwickelt wird nur die inzwischen flächendeckende Einführung von Globalhaushalten und Systemen der leistungsbezogenen Mittelvergabe bzw. die Nutzung von Zielvereinbarungen gesehen. Bei staatlich vorgegebenen Stellenplänen und detaillierten Vorgaben über die Größe und Struktur akademischer Teileinheiten durch Landesgesetz, kann eine Universitätsleitung jedoch kaum strukturbildend wirken.

Das in den Universitäten ,gefühlte” Ausmaß an tatsächlich zugewiesener Entscheidungsautonomie bleibt also weit hinter den Versprechungen der Hochschulpolitik zurück. Natürlich werden die Eingriffe seitens des Staates begründet: Mitentscheidung in der Studiengangentwicklung soll dem Zweck dienen, in einem sich entwickelnden Wettbewerb um Studierende die Vergleichbarkeit von Inhalten und Abschlüssen zu erhalten. ${ }^{16}$ Meist durch Vergleich mit ausländischen Spitzenuniversitäten belegt, sollen die Eingriffe in die Leitungs- und Organisationsstruktur die deutschen Universitäten für den internationalen Wettbewerb „fit” machen. ${ }^{17}$ Seitens der Universitäten wird im Gegenzug ebenso regelmäßig argumentiert, dass mit der Umsetzung der staatlichen Vorgaben neue Kosten verbunden sind, die, wenn nicht durch zusätzliche Finanzzuweisungen aufgefangen, diese Wettbewerbsfähigkeit gerade gefährden.

Unseres Wissens keine Aufmerksamkeit hat bisher jedoch das Argument erhalten, dass die mit der „Professionalisierung” einhergehende organisatorische Veränderung des Universitätswesens in Verbindung mit der Tatsache, dass Haushalte zunehmend verhandelt werden oder Zuweisungen aufgrund verhandelter Indikatorensysteme vorgenommen werden, allein und unmittelbar bereits Verluste an akademischer Leistungsfähigkeit und gestiegene Verwaltungskosten verursacht. Dies liegt ausschließlich darin begründet, dass an der Prinzipal-Agenten-Struktur im Verhältnis von Staat und Universität grundsätzlich fest gehalten wird. Verhandlungslösungen reflektieren aber das Bestreben der Parteien einen möglichst großen Rentenanteil zu erlangen. Schließlich kann sich der Staat dann sogar gedrängt sehen, auf die Zu-

\footnotetext{
${ }^{16}$ Tatsächlich zeigt Warning (2004), dass deutsche Universitäten strategische Differenzierungen bisher nur über die Wahl der Forschungsintensität gesucht haben.

${ }^{17}$ Die Pressemitteilung des Ministeriums für Wissenschaft und Kunst des Landes BadenWürttemberg zu seinem neuen Hochschulgesetz [MWK (2004)] ist diesbezüglich sehr charakteristisch. Nach entsprechenden einführenden Erläuterungen werden im Detail dann sehr tiefe gesetzliche Eingriffe in die Organisationsfreiheit der Universitäten dargestellt.
} 
nahme der Ineffizienz durch weitere Beschneidungen der Universitätsautonomie zu reagieren.

\section{Autonomie als Verhandlungsmacht: eine ein- fache Analyse}

\subsection{Der Modellrahmen}

\subsubsection{Wissenschaftliche Leistung und Performanzmessung}

Vereinfachend werden wir drei repräsentative Akteure betrachten. Das Wissenschaftsministerium ist an der Erstellung eines möglichst großen wissenschaftlichen Ertrags der Universität bei Minimierung der Kosten der Leistungserstellung interessiert. Es übernimmt die Rolle des Prinzipals. Im Folgenden entspricht der wissenschaftliche Ertrag der Leistung $e \geqq 0$, die von der Universität - repräsentiert durch ihre akademische Leitung - erbracht wird. Die akademische Leistungserstellung verursacht Kosten $c(e)$, mit $c(0)=c^{\prime}(0)=0, c^{\prime}(e)>0, c^{\prime \prime}(e)$ sowie, nur zur Vereinfachung, $c^{\prime \prime \prime}(e) \geqq 0$ für $e>0$ und $\lim _{e \rightarrow \infty} c(e)=\infty$. Die Wahl $e>0$ muss also als eine über die Normalleistung zur Aufrechterhaltung eines Regelbetriebs hinausgehende Leistung interpretiert werden. Dies erlaubt es, von den (fixen) Kosten dieser Normalleistung zu abstrahieren.

Da der wissenschaftliche Ertrag der Universität nicht marktfähig ist, muss der Prinzipal „Ministerium” seinen Agenten „Akademische Universitätsleitung” ein Budget $B$ für Forschung und Lehre zuweisen. Der Ertrag mag dabei für alle Parteien beobachtbar sein, er ist jedoch im Rahmen von Budgetverhandlung nicht direkt verifizierbar. ${ }^{18}$ Ein zweiter Agent - die „Universitätsverwaltung” - produziert jedoch

\footnotetext{
${ }^{18}$ Für die nachfolgende Analyse ist tatsächlich unerheblich, ob das Ministerium oder die Universitätsleitung den Ertrag beobachten können. Beide müssen nur eine unverzerrte Einschätzung dieses Ertrags vornehmen können, die allerdings, ohne auf die durch die Verwaltung produzierten Performanzsignale Bezug zu nehmen, nicht verifizierbar ist.
} 
ein verifizierbares Signal

$$
y=\left\{\begin{array}{l}
e, \text { mit Wahrscheinlichkeit } p(a) \\
0, \text { mit Wahrscheinlichkeit }(1-p(a))
\end{array}\right.
$$

wobei $a \geqq 0$ den Leistungseinsatz der Verwaltung in der Erstellung solcher Steuerungsinformation bezeichnet. Für die Signalproduktion gilt: $p(0)=p_{\min }>0$ und $p^{\prime}(a)>0$ sowie $p^{\prime \prime}(a) \leqq 0$ für $a \geqq 0$. Dabei impliziert $p_{\min }>0$, dass bereits die Erfüllung der Dienstpflichten zur Wahrung eines ordnungsgemäßen Studien- und Forschungsbetriebs informative und verifizierbare Signale über die akademische Leistungserstellung entstehen lässt.

Es handelt es sich hier um den Katalog deskriptiver Kennziffern wie Studierendenzahlen, Absolventenzahlen, Drittmittelsummen etc. Sollen darüber hinaus weitere Signale erstellt werden bzw. eine über die Deskription eines Zustandes hinausgehende Evaluation statt finden, muss die Verwaltung zusätzliche Informationsleistungen erbringen. Die spezielle funktionale Form (1) reflektiert dabei, dass sich die betrachtete Universität in einem Rang-Wettbewerb um Budgetmittel mit anderen Universitäten befindet. Die Wahrscheinlichkeit $p(a)$ bezeichnet also die Wahrscheinlichkeit in diesem Wettbewerb eine leistungsbezogene Mittelzuweisung zu erhalten. Wiederum wird von den (fixen) Verwaltungskosten eines Regelbetriebs abstrahiert, so dass nur die variablen Kosten der Produktion zusätzlicher Steuerungssignale betrachtet werden. Diese sind durch $k(a)$ gegeben, mit $k(0)=k^{\prime}(0)=0, k^{\prime}(a)>0$, $k^{\prime \prime}(a)$ sowie, nur zur Vereinfachung, $k^{\prime \prime \prime}(a) \geqq 0$ für $a>0$ und $\lim _{a \rightarrow \infty} k(a)=\infty$.

Zwei Formen der organisatorischen Zuordnung des Agenten „Verwaltung” werden im Folgenden unterschieden:

- In der „klassischen” Struktur der Universitätsverwaltung als Teil der Landesverwaltung ist diese dem Ministerium direkt unterstellt, berichtet ihm und wird von ihm mittels eines prädeteriminierten Systems der Leistungszulagen und Bewährungsaufstiege geführt.

- Im Zuge der Professionalisierung der Verwaltung wird sie in einen Agenten der Universitätsleitung umgewandelt, die zwar, da die Verwaltung Teil des öffentlichen Dienstes bleibt, das gleiche System der Performanzbewertung zum Zweck der Gewährung von Zulagen und Beförderungen einsetzen muss, aber 
durch Zuweisung eigener Budgetmittel und die Umwidmung von Stellen die Anreizintensität steuern kann.

Das durch Vereinbarungen zwischen den Tarifparteien entstandene System der Performanzbeurteilung für öffentliche Verwaltungstätigkeiten kann weder durch das Wissenschaftsministerium noch durch Universitätsleitung beeinflusst werden. Seine Anwendung im Rahmen eines Zulagen- und Beförderungssystems lässt Signale

$$
x=\left\{\begin{array}{l}
a, \text { mit Wahrscheinlichkeit } q \\
0, \text { mit Wahrscheinlichkeit }(1-q)
\end{array}\right.
$$

entstehen, wobei die Wahrscheinlichkeit $q$, eine überdurchschnittliche Leistung tatsächlich dokumentieren zu können, dann also exogen ist.

\subsubsection{Das System der Budgetzuweisungen und Verhandlungen}

Der Prinzipal „Ministerium” verhandelt mit seinem Agenten „Universität” vor Beginn einer Zuweisungsperiode über eine Budgetregel

$$
B(y)=S+b y,
$$

wobei $S$ den sogenannten Sockelbetrag und by den über eine leistungsbezogene Mittelvergabe zugewiesenen Betrag bezeichnet. Tatsächlich gibt es derzeit sowohl Verhandlungen über Zielvereinbarungen als auch Systeme, in denen die Mittelvergabe gemäß festgelegter Formeln statt findet. Dieser vor allem länderspezifische Unterschied kann aber nachfolgend für die Zwecke dieser Analyse vernachlässigt werden. Die auf Formeln beruhenden Zuweisungssysteme sind ebenfalls ursprünglich verhandelt worden und werden kontinuierlich nachverhandelt.

Alle in Deutschland zur Zeit etablierten Systeme leistungsbezogener Mittelvergaben sind mit Bestandsgarantien ausgestattet. D. h., die zugewiesenen Mittel schwanken zwar in Folge des Performanzwettbewerbs der Universitäten, können aber eine untere Grenze nicht unterschreiten. Zur Vereinfachung wird diese Grenze im Folgenden gleich Null gesetzt, so dass

$$
\begin{aligned}
S & \geqq 0, \\
S+b y & \geqq 0, \forall y
\end{aligned}
$$


erfüllt sein müssen. Im klassischen Fall der vom Prinzipal „Ministerium” direkt geführten Verwaltung übernimmt dieser auch die Kosten, die durch diese Aufgabenerfüllung entstehen. D. h., er weist der Universität Beförderungsstellen und Zulagenmittel in einem für seine Zwecke ausreichenden Maße ebenfalls direkt zu. In diesem Fall wird die Universität in den Verhandlungen mit dem Ministerium ihren Überschuss

$$
\pi^{K}(e, S, b) \equiv S+E_{y}\{b y\}-c(e)
$$

zu maximieren suchen.

Die für die berichterstellende Leistung der Verwaltung zu zahlende Kompensation wird als

$$
A(x)=F+d x
$$

berechnet. Da diese Leistungserstellung vor- und insgesamt ausfinanziert sein muss, gilt, unabhängig davon, ob das Ministerium oder die akademische Universitätsleitung als Prinzipal der Verwaltung in Erscheinung tritt,

$$
\begin{aligned}
F & \geqq 0, \\
F+d x & \geqq 0, \forall x .
\end{aligned}
$$

Allerdings ändert sich im Fall der Verwaltung als Agenten der Universitätsleitung deren Zielfunktion, die nun durch

$$
\pi^{P}(e, a, S, b, F, d) \equiv S+E_{y}\{b y\}-c(e)-F-E_{x}\{d x\}
$$

gegeben ist.

Die Verhandlungen zwischen Ministerium und Universität werden im Folgenden als Nash-Verhandlungsspiel modelliert, wobei ersteres im klassischen Fall der direkten, eigenen Führung der Hochschulverwaltung den erwarteten Nettoertrag der wissenschaftlichen Leistung

$$
\psi^{K}(e, a, S, b, F, d) \equiv e-S-E_{y}\{b y\}-F-E_{x}\{d x\}
$$

bzw. im Fall der in die Universität als Agenten integrierten Verwaltung

$$
\psi^{P}(e, S, b) \equiv e-S+E_{y}\{b y\}
$$

zu maximieren sucht. Um den Vergleich möglichst „reiner” Organisationsregime zu ermöglichen, schließen wir für den Fall der klassischen Verwaltung aus, dass diese 
mit der Universitätsleitung kooperiert und dadurch bereits ein Delegationsverhältnis zwischen diesen beiden Akteuren etabliert wird.

Die Verhandlungsmacht $\gamma \in(0,1)$ der Universität spiegelt den Grad ihrer Entscheidungsautonomie wieder. Existierten z. B. so viele gesetzliche Eingriffe und Verordnungen, dass die Universitätsleitung in der Wahl ihrer Organisation vollständig festgelegt wäre, könnte sie in den Budgetverhandlungen auch keinen Spielraum geltend machen, in den das Ministerium durch Verhandlungen über eine leistungsbezogene Mittelvergabe zusätzlich zu diesen gesetzlichen Regelungen steuernd eingreifen müsste. Erst ein weniger „perfektes” System von gesetzlichen Vorgaben und Verordnungen lässt einen solchen Spielraum entstehen und schafft dadurch gleichsam die Notwendigkeit der Steuerung über Zuweisungen. Die Verhandlungslösung maximiert dann das Nash-Produkt. ${ }^{19}$

$$
\Omega^{V}=\left(\pi^{V}\right)^{\gamma}\left(\psi^{V}\right)^{(1-\gamma)}
$$

für die beiden Fälle $V \in\{K, P\}$.

Schließlich kann die Universität im Fall der professionalisierten Verwaltung interne Mittel im Rahmen eines Globalhaushaltsregime zuweisen. Dies impliziert die (zwar eingeschränkte) Möglichkeit, Mittel über Haushaltsjahre zu verteilen. Zur Vereinfachung, unterstellen wir deshalb, dass

$$
S+E_{y}\{b y\}-F-E_{x}\{d x\} \geqq 0
$$

als Bedingung für eine ausgeglichene Haushaltsführung im Fall des von der Universität selbst auszustattenden, professionalisierten Hochschulmanagements. ${ }^{20}$

\subsubsection{Die Signalproduktion der Verwaltung}

Die Verwaltung richtet sich in ihrer Leistungserstellung grundsätzlich an dem für sie existierenden System der Performanzmessung aus. D. h., sie maximiert ihren erwarteten Ertrag

$$
F+q d a-k(a)
$$

\footnotetext{
${ }^{19}$ Die nachfolgende Analyse dieser Lösung erfolgt in Anlehnung an Demougin/Helm (2005).

${ }^{20}$ Im Fall der klassischen Verwaltung können keine Mittel zwischen Verwaltung und akademischen Bereich umgeschichtet werden.
} 
durch Wahl des Leistungsniveaus $a$. Die notwendige Bedingung

$$
q d-k^{\prime}(a)=0
$$

charakterisiert eine optimale Leistung

$$
a^{*}(d ; q)=\left\{\begin{array}{l}
k^{\prime^{-1}}(d q), \text { wenn } d>0 \\
0, \text { wenn } d \leqq 0
\end{array}\right.
$$

Dies impliziert, dass nur zwei Konstellationen möglich sind. Entweder der Prinzipal der Verwaltung - das Ministerium bzw. die akademische Universitätsleitung - sind an einer positiven Leistung in der Erstellung von Steuerungssignalen interessiert und wählen daher eine Anreizintensität $d^{V}>0, V \in\{K, P\}$. Oder sie sind nicht an einer solchen Anstrengung interessiert und wählen konsequenterweise $d^{V}=0$.

Da das Performanz-Evaluationsverfahren in der Verwaltung exogen gegeben ist, beschreibt also (17) eine für beide potentiellen Prinzipale geltende Relation, die durch die Wahl der organisationsspezifischen Anreizintensität $d^{V}$ gestaltet werden kann. Die Annahmen über die Kostenfunktion $k(a)$ implizieren weiterhin, dass

$$
\begin{gathered}
E_{X}\left\{F^{V}+d^{V} x-k\left(a^{*}\left(d^{V} ; q\right)\right) \mid a^{*}\left(d^{V} ; q\right)\right\} \\
=F^{V}+k^{\prime}\left(a^{*}\left(d^{V} ; q\right)\right) a^{*}\left(d^{V} ; q\right)-k\left(a^{*}\left(d^{V} ; q\right)\right) \geqq 0,
\end{gathered}
$$

mit strikter Ungleichheit wenn $d^{V}>0$. Weder der (10) maximierende Prinzipal „Universitätsleitung” noch der Prinzipal „Ministerium” mit der Zielfunktion (11) werden daher in ihrem Optimum eine positive performanzunabhängige Zahlung $F$ bieten. Eine solche Zahlung besitzt keinerlei Anreizwirkungen und ist, gegeben (16), auch nicht notwendig um eine Partizipationsbedingung zu erfüllen. Es gilt also $F^{V *}=0$ für $V \in\{K, P\}$.

Die Argumente lassen sich in Form einer Minimalkostenfunktion für die Signalproducktion der Verwaltung zusammen fassen:

$$
K^{V}\left(d^{V}\right)=k^{\prime}\left(a^{*}\left(d^{V}\right)\right) a^{*}\left(d^{V}\right) \geqq 0, V \in\{K, P\},
$$

mit strikter Ungleichheit wenn $d^{V}>0$. Gegeben die Annahmen über die Kostenfunktion $k(a)$, gilt offensichtlich $K\left(a^{*}\right)-k\left(a^{*}\right) \geqq 0$. D. h., im Fall $d^{V}>0$ erhält die Verwaltung immer eine positive Rente. 
Ein letztes Mal sei darauf hingewiesen, dass in der Herleitung von (19) von allen Kosten der Aufrechterhaltung eines Regelbetriebs durch die Verwaltung abstrahiert worden ist. Die Kostenfunktion erfasst ausschließlich die zusätzlichen Kosten, die durch die Produktion von Steuerungssignalen entstehen. Dies impliziert eine sehr enge Sicht der Problemlage, die bewusst in Kauf genommen wird, um die Kostenund Leistungseffekte identifizieren zu können, die allein durch die organisationelle Veränderung entstehen. Aus dem gleichen Grund werden mögliche Effizienzgewinne, die in Folge der Einführung von Globalhaushalten und Prozessorientierung im Management der Universität entstehen können, nicht berücksichtigt.

\subsection{Der Fall der klassischen Universitätsverwaltung}

Gegeben eine Wahl der Anreizintensität $d^{K}$ durch das Ministerium, können beide der Prinzipal und der Agent „Universitätsleitung” - die Wahrscheinlichkeit $p\left(a^{*}\left(d^{K *}\right)\right)$ $>0$, mit der ein Leistungsausweis des Agenten ex-post dokumentiert wird, in ihren bilateralen Verhandlungen korrekt antizipieren. Anreizkompatibilität für die Universitätsleitung impliziert dann

$$
e^{K}=\arg \max _{\hat{e} \geq 0} S^{K}+E_{y}\left\{b^{K} y\right\}-c(\hat{e})
$$

und die entsprechende charakteristische Bedingung erster Ordnung

$$
b^{K} p\left(a^{*}\left(d^{K}\right)\right)-c^{\prime}\left(e^{K}\right)=0
$$

wobei das Superskript „K” wieder den betrachteten Organisationsfall markiert.

Daher gilt, wegen (4),

$$
S^{K}+b^{K} y=\left\{\begin{array}{c}
S^{K}+c^{\prime}\left(e^{K}\right) e^{K} \\
S^{K}
\end{array}\right\} \geqq 0
$$

wobei die erste Zeile strikt positiv ist, wenn die Universität eine wissenschaftliche Leistung $e^{K}>0$ tatsächlich erbringen soll. Die Nebenbedingung (5) kann also für die Verhandlungen zwischen Universität und Ministerium nicht bindend wirken. Nur die Nicht-Negativitätsbedingung (4) für den Sockelbetrag $S$ und die Anreizkompatibilitätsbedingung (21) müssen also als Restriktionen für das Verhandlungsproblem (13), mit $V=K$, berücksichtigt werden. 
Durch Einsetzen aus (21) entsteht so das Lagrange-Optimierungsproblem

$$
\begin{aligned}
\max _{\left(e^{K}, S^{K}, \lambda^{K}\right)} \mathcal{L}^{K}= & \gamma \ln \left(S^{K}+c^{\prime}\left(e^{K}\right) e^{K}-c\left(e^{K}\right)\right) \\
& +(1-\gamma) \ln \left(e^{K}-S^{K}-c^{\prime}\left(e^{K}\right) e^{K}-K^{K}\left(d^{K}\right)\right)-\lambda^{K} S^{K} .
\end{aligned}
$$

Die entsprechenden notwendigen Bedingungen lauten:

$$
\begin{gathered}
\gamma \frac{c^{\prime \prime}\left(e^{K *}\right) e^{K *}}{S^{K *}+c^{\prime}\left(e^{K *}\right) e^{K *}-c\left(e^{K *}\right)} \\
+(1-\gamma) \frac{1-c^{\prime \prime}\left(e^{K *}\right) e^{K *}-c^{\prime}\left(e^{K *}\right)}{e^{K *}-S^{K *}-c^{\prime}\left(e^{K *}\right) e^{K *}-K^{K}\left(d^{K}\right)}=0 \\
-(1-\gamma) \frac{1}{e^{K *}-S^{K *}-c^{\prime}\left(e^{K *}\right) e^{K *}-K^{K}\left(d^{K}\right)}-\lambda^{K *}=0
\end{gathered}
$$

Wenn ein positiver Sockelbetrag $\left(S^{K *}>0\right)$ vereinbart wird, lässt sich (24) mit Hilfe von (25) in die Bedingung

$$
1-c^{\prime}\left(e^{K *}\right)=0
$$

umformen, so dass in diesem Fall das Leistungsniveau der Universität dem erstbesten entspricht: $e^{K *}=c^{\prime-1}(1) \equiv e^{F B}$. Aus (25) folgt dann weiterhin

$$
\begin{aligned}
\gamma & =\frac{S^{K *}}{e^{K *}-c\left(e^{K *}\right)-K^{K}\left(d^{K}\right)}+\frac{c^{\prime}\left(e^{K *}\right) e^{K *}-c\left(e^{K *}\right)}{e^{K *}-c\left(e^{K *}\right)-K^{K}\left(d^{K}\right)} \\
& =\frac{S^{K *}}{e^{F B}-c\left(e^{F B}\right)-K^{K}\left(d^{K}\right)}+\frac{e^{F B}-c\left(e^{F B}\right)}{e^{F B}-c\left(e^{F B}\right)-K^{K}\left(d^{K}\right)}
\end{aligned}
$$

Die Bedingung (27) und $\gamma \in(0,1)$ implizieren daher, dass die Lösung $S^{K *}>0$ für alle Leistungsintensitäten der Verwaltung $d^{K} \geqq 0$ ausgeschlossen ist.

Wenn aber die Nicht-Negativitätsbedingung für den Sockelbetrag bindet, ist das optimale Leistungsniveau der Universität durch die Anreizkompatibilitätsbedingung (21) determiniert:

$$
e^{K^{*}}=c^{\prime-1}\left(b^{K} p\left(a^{*}\left(d^{K}\right)\right)\right) .
$$

Es gilt dann $1-c^{\prime}\left(e^{K *}\right)>0-$ d. h., es wird ein zweitbestes Niveau realisiert. Im Ergebnis der Budgetverhandlungen erhält also der Agent „Universitätsleitung” die positive Rente $b^{K} p\left(a^{*}\left(d^{K}\right)\right) e^{K *}-c\left(e^{K *}\right)=c^{\prime}\left(e^{K *}\right) e^{K *}-c\left(e^{K *}\right)>0$. Aus (24) 
folgt dann auch, dass $\partial e^{K^{*}} / \partial \gamma>0$ : Universitätsleitungen mit größerer Verhandlungsmacht können einen höhere Rente abschöpfen. Diese höhere Rente wird durch stärkere Bemühungen um wissenschaftlichen Ertrag induziert.

Der rationale Prinzipal "Ministerium" wird den Effekt seiner Wahl der Anreizintensität $d^{K}$ für die Verwaltung auf die Verhandlungen antizipieren. Das entsprechende ex-ante Optimierungsproblem des Ministeriums lässt sich also durch

$$
\begin{aligned}
\max _{d^{K}} \Psi^{K *}\left(d^{K}\right)= & e^{K^{*}}\left(d^{K}\right)-c^{\prime}\left(e^{K^{*}}\left(d^{K}\right)\right) e^{K^{*}}\left(d^{K}\right)-K^{K}\left(d^{K}\right) \\
\text { u. d. N.: } & \quad d^{K} \geqq 0
\end{aligned}
$$

angeben, wobei $e^{K^{*}}\left(d^{K}\right)=e^{K^{*}}\left(a^{*}\left(d^{K}\right)\right.$ durch (28) und (17) gegeben ist. Wird die Nebenbedingung (29) zunächst vernachlässigt, wäre die entsprechende innere Lösung durch

$$
\begin{gathered}
{\left[\left[1-c^{\prime}\left(e^{K^{*}}\left(a^{*}\left(d^{K *}\right)\right)\right)-c^{\prime \prime}\left(e^{K^{*}}\left(a^{*}\left(d^{K *}\right)\right)\right) e^{K^{*}}\left(a^{*}\left(d^{K *}\right)\right)\right] \frac{\partial e^{K^{*}}}{\partial a^{*}}\right.} \\
\left.-k^{\prime \prime}\left(a^{*}\left(d^{K *}\right)\right) a^{*}\left(d^{K *}\right)-k^{\prime}\left(a^{*}\left(d^{K *}\right)\right)\right] \frac{\partial a^{*}\left(d^{K *}\right)}{\partial d^{K}}=0
\end{gathered}
$$

charakterisiert. Da $\frac{\partial e^{K^{*}}}{\partial a^{*}}>0$ und $\frac{\partial a^{*}\left(d^{K *}\right)}{\partial d^{K}}>0$, folgt jedoch aus (24), dass der Ausdruck in (30) kleiner Null sein muss. D. h., die Nebenbedingung des Optimierungsproblems (29) bindet: $d^{K *}=0$. Es folgt, dass $a^{*}\left(d^{K *}\right)=0, p\left(a^{*}\left(d^{K *}\right)\right)=p_{\min }>0$ und $K^{K}\left(d^{K *}\right)=0$. Die notwendigen Bedingungen (21) und (24) determinieren schließlich eindeutig $e^{K *}$ und $b^{K *}$.

In der klassischen Organisation der Universitätsverwaltung als Teil der allgemeinen Landesverwaltung investiert ein rationaler Prinzipal „Ministerium” also nie in die Produktion von Steuerungssignalen. Solche Investitionen sind aus seiner Sicht nur mit direkten Kosten $K^{K}(d)$ und weiteren indirekten Kosten verbunden, die sich, bei gegebenem Volumen der leistungsbezogenen Mittelvergabe $b^{K}$, in einer höheren Wahrscheinlichkeit der Zuweisung nieder schlagen. Der reine Anreizeffekt auf die Universitätsleitung ist aber kostenneutral auch durch ein höheres Volumen $b^{K}$ bei geringerer Auszahlungswahrscheinlichkeit erreichbar und die tatsächlich zu tragenden Kosten der wissenschaftlichen Leistungserstellung sind unabhängig davon allein durch die Rente der Universität determiniert. Letztere hängt aber nicht von den Aktivitäten der Verwaltung, sondern nur vom (implizit) verhandelten wissenschaftlichen Leistungsniveau $e^{K *}$ ab. 


\subsection{Der Fall der ,professionalisierten” Verwaltung}

Die Professionalisierung der Verwaltung führt zu einem Prozess, in dem die bisherige alleinige Aufgabe der Aufrechterhaltung eines ordnungsgemäßen Betriebes zunehmend in eine Aufgabe des Managements von Universitätsinteressen umgewandelt wird. Gleichzeitig erfolgt damit eine Unterstellung dieses Managements als Agenten der Universitätsleitung. So werden auch die Verhandlungspositionen von Ministerium und Universität verändert. Budgetverhandlungen sind nun mit einem Agenten „Universitätsleitung” zu führen, der ein Eigeninteresse an der Darstellung seiner wissenschaftlichen Leistungen besitzt und über die Möglichkeiten verfügt, diese Darstellung zu beeinflussen.

Die Universitätsleitung wählt nun zwei Handlungen $a$ und $e$, um ihren Erwartungsnutzen zu maximieren. Anstelle der alten Anreizkompatibilitätsbedingung (20) gilt also:

$$
\left(d^{P}, e^{P}\right)=\arg \max _{\hat{d} \geq 0, \hat{e} \geq 0} S+E_{y}\{b y\}-c(\hat{e})-K\left(a^{*}(\hat{d})\right)
$$

Die beiden notwendigen Bedingungen lauten:

$$
\begin{aligned}
b p\left(a^{*}\left(d^{P}\right)\right)-c^{\prime}\left(e^{P}\right) & =0 \\
{\left[b e^{P} p^{\prime}\left(a^{*}\left(d^{P}\right)\right)-K^{\prime}\left(a^{*}\left(d^{P}\right)\right)\right] \frac{\partial a^{*}\left(d^{P}\right)}{\partial d} } & =0
\end{aligned}
$$

Aufgrund der Eigenschaften der Kostenfunktion $k(a)$ ist leicht zu sehen, dass (33) für $e^{P}>(=) 0$ nun $a^{*}\left(d^{P}\right)>(=) 0 \rightarrow d^{P}>(=) 0$ impliziert. Wird aus (32) in (33) für den Bonus $b$ eingesetzt, folgt offensichtlich

$$
e^{P} c^{\prime}\left(e^{P}\right)=p\left(a^{*}\left(d^{P}\right)\right) k^{\prime}\left(a^{*}\left(d^{P}\right)\right)
$$

Abbildung 1 zeigt dann, dass die Zuweisung der Verantwortlichkeit für die Signalproduktion an den produktiven Agenten „Universität” immer dafür sorgt, dass dieser auch in die unproduktive Informationsaktivität investiert.

\section{Abbildung 1 hier einfügen!}

Da die Signalproduktion der Verwaltung intuitiv den Wunsch der Universitätsleitung reflektiert, eine wissenschaftliche Leistung $e^{P}$ besser darzustellen, bietet es 
sich an, aus (32) und (33) die Funktion $a^{P *}\left(e^{P}\right)$ zu definieren. Diese Funktion erfüllt, wie oben bereits diskutiert,

$$
\begin{gathered}
\frac{\partial a^{P *}\left(e^{P}\right)}{\partial e^{P}}=\frac{1}{p^{\prime}\left(a^{P *}\right) c^{\prime \prime}\left(e^{P}\right)}\left[p^{\prime}\left(a^{P *}\right) K^{\prime}\left(a^{P *}\right)+p\left(a^{P *}\right) K^{\prime \prime}\left(a^{P *}\right)\right. \\
\left.-\frac{p^{\prime \prime}\left(a^{P *}\right) p\left(a^{P *}\right) K^{\prime}\left(a^{P *}\right)}{p^{\prime}\left(a^{P *}\right)}\right]>0
\end{gathered}
$$

Gegeben (32) und (4), kann die Nebenbedingung (5) für die Maximierung von (13), für $V=P$, nicht bindend wirken. Das Verhandlungsproblem ist also in diesem Fall durch

$$
\begin{aligned}
\max _{\left(e^{P}, S^{P}, \lambda^{P}\right)} \mathcal{L}^{P}= & \gamma \ln \left(S^{P}+c^{\prime}\left(e^{P}\right) e^{P}-c\left(e^{P}\right)-K^{P}\left(a^{P *}\left(e^{P}\right)\right)\right) \\
& +(1-\gamma) \ln \left(e^{P}-S^{P}-c^{\prime}\left(e^{P}\right) e^{P}\right)-\lambda^{P} S^{P}
\end{aligned}
$$

gegeben. Da $c\left(e^{P}\right) \geqq 0$, ist, gegeben (4) und (5), auch unmittelbar klar, dass die Lösung des Problems (36) die Bedingung für eine ausgeglichene Haushaltsführung (14) immer mit strikter Ungleichheit erfüllen wird. Die Bedingungen erster Ordnung lauten daher:

$$
\begin{gathered}
\gamma \frac{c^{\prime \prime}\left(e^{P *}\right) e^{P *}-K^{\prime}\left(a^{P *}\left(e^{P *}\right)\right) \frac{\partial a^{P *}\left(e^{P *}\right)}{\partial e^{P}}}{S^{P *}+c^{\prime}\left(e^{P *}\right) e^{P}-c\left(e^{P *}\right)-K^{P}\left(a^{P *}\left(e^{P *}\right)\right)} \\
+(1-\gamma) \frac{1-c^{\prime \prime}\left(e^{P *}\right) e^{P *}-c^{\prime}\left(e^{P *}\right)}{e^{P *}-S^{P *}-c^{\prime}\left(e^{P *}\right) e^{P *}}=0 \\
\gamma \frac{1}{S^{P *}+c^{\prime}\left(e^{P *}\right) e^{P}-c\left(e^{P *}\right)-K^{P}\left(a^{P *}\left(e^{P *}\right)\right)} \\
-(1-\gamma) \frac{1}{e^{P *}-S^{P *}-c^{\prime}\left(e^{P *}\right) e^{P *}}-\lambda^{P *}=0
\end{gathered}
$$

Für $\lambda^{P *} \neq 0 \rightarrow S^{P *}=0$ folgt aus (36) wieder $\partial e^{K^{*}} / \partial \gamma>0$. Universitätsleitungen mit größerer Verhandlungsmacht schöpfen auch in diesem Fall eine höhere Rente durch stärkere Bemühungen um wissenschaftlichen Ertrag ab. Allerdings investieren sie immer einen Teil der zugewiesenen Ressourcen in die unproduktive Berichterstattung, auf deren Basis die leistungsbezogenen Mittel verteilt werden. D. h., es wird mit höherer Wahrscheinlichkeit ein geringerer wissenschaftlicher Ertrag ausgewiesen.

Ist dies aus Sicht eines offensichtlich innovationsfreudigen - und vieleicht auch durchaus wohlmeinenden - Prinzipals „Ministerium” nicht schon enttäuschend genug, 
entsteht in Folge der Organisationsreform sogar ein qualitativ völlig neuartiges und noch enttäuschenderes Verhandlungsregime. Es sei $\bar{e}^{P}$ definiert durch

$$
1-c^{\prime}\left(\bar{e}^{P}\right)-K^{\prime}\left(a^{P *}\left(\bar{e}^{P}\right)\right) \frac{\partial a^{P *}\left(\bar{e}^{P}\right)}{\partial e^{P}}=0
$$

und

$$
\gamma^{P} \equiv \frac{c^{\prime}\left(\bar{e}^{P}\right) \bar{e}^{P}-c\left(\bar{e}^{P}\right)-K\left(a^{P *}\left(\bar{e}^{P}\right)\right)}{\bar{e}^{P}-c\left(\bar{e}^{P}\right)}
$$

der Rentenanteil des Agenten „Universitätsleitung” bei Wahl von $e^{P *}=\bar{e}^{P}$. Wegen (37) gilt dann für alle $\gamma \geqq \gamma^{P}$, dass die Nicht-Negativitätsbedingung für den Sockelbetrag $S^{P}$ (4) nicht mehr bindend wirkt: $\lambda^{P *}=0, \forall \gamma \geqq \gamma^{P}$. Alle Universitäten mit einer Verhandlungsmacht $\gamma \geqq \gamma^{P}$ wählen - gemäß (37) und (38) - also das gleiche Leistungsniveau $e^{P *}=\bar{e}^{P}<e^{F B}$. In diesem Leistungsregime führt eine größere Verhandlungsmacht der Universität nur zu einem höheren positiven Sockelbetrag. Der Sockelbetrag wird zum alleinigen Instrument zur Abschöpfung höheren Renteneinkommens durch die Universität.

\subsection{Interpretation der Analyse}

Es ist für die vorgeführte Analyse unerheblich, aus welchen Gründen die „Professionalisierung" der Verwaltung tatsächlich angestrebt wird. Aus informationsökonomsicher Sicht ist nur wichtig zu erkennen, dass sich mit der Unterstellung der Verwaltung unter die akademische Universitätsleitung und die damit verbundene Veränderung des Berichtswesens über wissenschaftliche Leistung auch die Verhandlungssituation zugunsten der Universität verändert wird. Diese kann die Umwidmung von Mitteln für Forschung und Lehre und sogar die Einforderung eines zusätzlichen Sokkelbetrags öffentlich sehr gut begründen. Schließlich ist sie doch nun gezwungen auch noch die Kosten des Controllings zu tragen und investiert nur zu dem Zweck, die Qualität dieses Systems zu verbessern.

Dennoch wird kaum zu vermeiden sein, dass sich die Enttäuschung des Prinzipals „Ministerium” in weiteren Eingriffshandlungen nieder schlagen wird. Insbesondere wenn sich Universitätsleitungen nun Sockelbeträge sichern können, liegt es für ein solches Ministerium auf der Hand, die Verhandlungsmacht der Universität zu beschneiden. Dies kann es aber nur tun, in dem es durch Gesetz und Verordnung die Autonomie der Hochschule in der Wahl ihrer internen Organisation einschränkt 
- speziell die Ausgestaltung des Controllings sowie, in dem Versuch Skaleneffekte zu generieren, die Anzahl und Größe der zu steuernden Einheiten vorgibt.

Selbst wenn es dem Ministerium dadurch gelingt, Kostenreduktionen in der Verwaltung in einem gewissen Ausmaß zu erzwingen, bleibt dieser Ansatz jedoch fehlgeleitet, da er die Gründe für die beschriebene Entwicklung verkennt. Er gefährdet sogar die Leistungsfähigkeit eines durchaus heterogenen Universitätssystems insgesamt. Gesetze und Verordnungen gelten prinzipiell für alle Universitäten in gleicher Weise. Die Verhandlungsmacht einer Universität hängt jedoch in einem nicht perfekt reguliertem System auch von der Fächerstruktur sowie regionalen und lokalen Besonderheiten ab, die sich z. B. in der Möglichkeit nieder schlagen, über politische Lobbyisten Einfluss auf den Prinzipal „Ministerium” auszuüben.

Der Versuch, die als übermäßig empfundene Verhandlungsmacht einzelner Universitäten (mit $\gamma \geqq \gamma^{P}$ ) durch Gesetz und Verordnung zu beschneiden, ohne einen Leistungsverlust riskieren zu müssen, greift damit auch beschränkend in die Entscheidungsautonomie solcher Universitäten ein, die gegenwärtig nur über eine geringe Verhandlungsmacht (mit $\gamma<\gamma^{P}$ ) verfügen. Diese Universitäten reagieren dann aber mit einer Reduktion ihrer wissenschaftlichen Leistung. Es entsteht also im System insgesamt ein Verlust an wissenschaftlichen Ertrag.

\section{Ausblick}

Die informationsökonomische Analyse dessen, was sich organisatorisch in Universitäten verändert hat, führt also zunächst nur zu einer Erklärung, warum die beiden Akteure „Ministerium” und „Universität” gegenseitig berechtigte Vorwürfe der Verschwendung von Ressourcen bzw. des Wortbruchs hinsichtlich der versprochene größeren Entscheidungsautonomie vorbringen können. Sie reflektieren eine Verengung des Blickwinkels allein auf Reformen der Universitätsverwaltung unter Vernachlässigung der weiteren Effekte dieser Reformen auf das bilaterale Interessenverhältnis. Da Ministerien letztlich dann doch über die zusätzliche Alternative der Eingriffsregulierung verfügen, um die Rahmenbedingungen von Verhandlungen zu gestalten, scheint der Weg noch tiefer hinein in die Sackgasse „ausufernder” Ministerialbürokratie unter Inkaufnahme von Verlusten in der Leistungsbereitschaft der 
Wissenschaftler vorgezeichnet und fast unausweichlich.

Selbst der Versuch der Aufklärung über die Strukturen und der Annäherung durch vertrauensbildende Maßnahmen, den der rational argumentierende Ökonom unternehmen würde, scheint wenig fruchtbar, da er die eingeleiteten Reformen kaum umkehren kann. Aber wohl auch nicht umkehren wollte - schließlich ist kaum bestreitbar, dass durch die Professionalisierung der Verwaltung Anreize zur effektiveren Aufgabenerfüllung im Forschungs- und Lehrbetrieb entstehen. Dennoch gibt es eine Lösung. Und diese Lösung ist in der zunächst harmlos anmutenden, weil jedem Versuch der Modellierung eines Prinzipal-Agenten-Verhältnisses von Hochschulpolitik und Universität zugrunde liegenden, Annahme angelegt, dass der Ertrag einer Universität grundsätzlich nicht marktfähig ist.

Diese Annahme ist offensichtlich falsch - zumindest bezogen auf den größeren Teil der Lehrleistungen und auch hinsichtlich der Forschungsleistung, soweit es sich nicht um Grundlagenforschung im engeren Sinn handelt. In anderen Teilen der Welt wird täglich belegt, dass diese Annahme keine „natürliche” Beschränkung der Organisation eines Universitätssystems darstellt. Das oben beschriebene Prinzipal-AgentenVerhältnis selbst, das nun höchst imperfekt z. B. durch Zielvereinbarungsmodelle „marktähnlich” gestaltet werden soll, ist also tatsächlich bereits Ergebnis einer verfehlten Hochschulpolitik. Würde man den Universitäten endlich das Recht zugestehen, vermarktbare Leistungen auch zu vermarkten, wäre der in Verhandlungen noch zu lösende Interessenkonflikt reduziert. Ein Interessenausgleich müsste nur noch für die Leistungen erreicht werden, die tatsächlich öffentliche Güter sind. Die Leistungselastizität von Performanzindikatoren für Grundlagenforschung im engeren Sinn und damit die Möglichkeiten, Renten zu generieren, dürften darüber hinaus gering sein.

Aus unserer Sicht ist eine solche Entwicklung nicht nur zu erhoffen, sondern auch zu erwarten. Der in der oben beschriebenen Sackgasse gegenseitiger Vorwürfe und neuerlicher Eingriffe in die Autonomie der Universitäten inzwischen erreichte Zustand ist für alle Beteiligte tatsächlich kaum noch tragbar und politisch nicht länger aufrecht zu erhalten. Alle Zeichen weisen auf eine grundlegende Reform der Wissenschaftsorganisation unter Einschluss von Studiengebühren und verstärkten „Public-Private-Partnerships” in der angewandten Forschung hin. Um neuerliche Fehlentwicklungen in der Regulierung der entstehenden Märkte für wissenschaftliche 
Leistungen zu vermeiden, gebietet dann die Erfahrung mit den Reformprojekten der zurück liegenden Jahre, diese bevorstehende Reform durch ökonomisch-analytisch kompetente Beratung professionell zu begleiten.

\section{$5 \quad$ Literaturverzeichnis}

Chwolka, Anne (1996). Controlling als ökonomische Institution - Eine agencytheoretische Analyse. Physica, Heidelberg.

Demougin, Dominique und Carsten Helm (2005). Moral Hazard and Bargaining Power. Diskussionspapier, Wirtschaftswissenschaftliche Fakultät, Humboldt Universität zu Berlin.

Fabel, Oliver und Frank Heße (1999). Befragungsstudie vs. Publikationsanalyse: Zur Interpretation von Ranglisten der Forschungsaktivitäten deutscher betriebswirtschaftlicher Fachbereiche. Die Betriebswirtschaft - DBW, 59. Jg., S. 196-204.

Fabel, Oliver und Bodo Hilgers (2001). Leistungsbezogene Besoldung für Professor(inn)en - Eine „Lehrbuch-Analyse” des BMBF Konzepts. In: BackesGellner, Uschi, Matthias Kräkel, Dieter Sadowski und Johannes Mure (Hrsg.), Entlohnung, Arbeitsorganisation und personalpolitische Regulierung, Hampp, Mering, S. 21-38.

Fabel, Oliver, Erik Lehmann und Susanne Warning (2002). Der relative Vorteil deutscher wirtschaftswissenschaftlicher Fachbereiche im Wettbewerb um studentischen Zuspruch. Schmalenbachs Zeitschrift für betriebswirtschaftliche Forschung, 54. Jg., S. 509 - 375.

Fabel, Oliver, Erik Lehmann und Susanne Warning (2003). Vorträge als Qualitätsindikatoren: Empirische Evidenz der Jahrestagungen des Vereins für Socialpolitik. In: Backes-Gellner, Uschi und Corinna Schmidtke (Hrsg.), Hochschulökonomie - Analyse interner Steuerungsprobleme und gesamtwirtschaftlicher Effekte, Duncker und Humblot, Reihe „Schriften des Vereins für Socialpolitik - Neue Folge", SVS 296, Berlin, S. 13-31. 
Fedrowitz, Jutta, Erhard Krasny und Frank Ziegele (1999). Hochschulen und Zielvereinbarungen - neue Perspektiven der Autonomie, Verlag Bertelsmann Stiftung, Gütersloh.

Geis, Max.Emanuel (2003). Das Selbstbestimmungsrecht der Universitäten. Forschung ES Lehre, 5/2003, S. 242 - 245.

Görke, Wolfgang (2003). Zentralisierung versus Autonomie? Forschung 6 Gehre, 2/2003, S. $73-76$.

Heise, Dietmar (2002). Manager als Hochschulleiter? Pro. Forschung $\&$ Lehre, 4/2002, S. 178.

Hochschulrektorenkonferenz (2004). Professionalisierung als Leitungsaufgabe. Entschließung des 202. Plenums der HRK am 08.06.2004, Bonn.

Hommelhoff, Peter (2002). Manager als Hochschulleiter? Contra. Forschung 6 Lehre, 4/2002, S. 179.

Johannes Gutenberg Universität Mainz (2001). Eine Hochschule im Aufbruch Das neue Steuerungsmodell, Mainz.

Kirchhof, Paul (2003). Universität zwischen Freiheit und Steuerung. Forschung E Lehre, 5/2003, S. 234 - 240.

Leszcensky, Michael (2003). Internes und externes Hochschulcontrolling. HISKurzinformation, A8/2003, Hannover.

Leszcensky, Michael und Dominic Orr (2004). Staatliche Hochschulfinanzierung durch indikatorgestützte Mittelverteilung: Dokumentation und Analyse der Verfahren in 11 Bundesländern. HIS-Kurzinformation A2/2004, Hannover.

Müller-Böling, Detlef (2000). Die entfesselte Hochschule. Verlag Bertelsmann Stiftung, Gütersloh.

MWK (2004). „Das modernste Hochschulrecht in Deutschland” - Frankenberg sieht in dem neuen Hochschulgesetz „Weg zu unternehmerischen Hochschulen geöffnet". Pressemitteilung des Ministeriums für Wissenschaft und Kunst, Baden-Württemberg, 9. Dezember 2004. 
Schiedermaier, Hartmut (1997). Autonomie im Widerspruch. In: Hollerith, Josef (Hrsg.): Leistungsfähige Hochschulen - Aber wie? Luchterhand, Reihe „Hochschulwesen, Wissenschaft und Praxis”, Neuwied, S. 15 - 23.

Schock, Thomas A. H. (2002). Ziele vergeben, Budgets bereit stellen. Forschung Es Lehre, 4/2002, S. 180 - 182.

Starbatty, Joachim (2004). „Sire, geben Sie Gestaltungsfreiheit”. Forschung 8 Lehre, 7/2004, S. 367 - 368.

Stifterverband für die deutsche Wirtschaft (2002). Qualität durch Wettbewerb und Autonomie: Landeshochschulgesetze im Vergleich. Reihe „Positionen”, Essen.

Warning, Susanne (2004). Performance Differences in German Higher Education: Empirical Analysis of Strategic Groups. Review of Industrial Organization, vol. 24, S. $393-408$.

Weiler, Hans N. (1998). Verantwortung und Zuständigkeit - Entscheidungsstrukturen im amerikanischen Hochschulsystem auf dem „Schlachtfeld des Systemvergleichs". In: Müller-Böling, Detlef und Jutta Fedrowitz (Hrsg.), Leitungsstrukturen für autonome Hochschulen, Verlag Bertelsmann Stiftung, Gütersloh, S. $47-58$. 
Abbildung 1: Der Zusammenhang zwischen wissenschaftlicher Leistungserstellung und informationsgenerierenden Aktivitäten bei professionalisierter Verwaltung

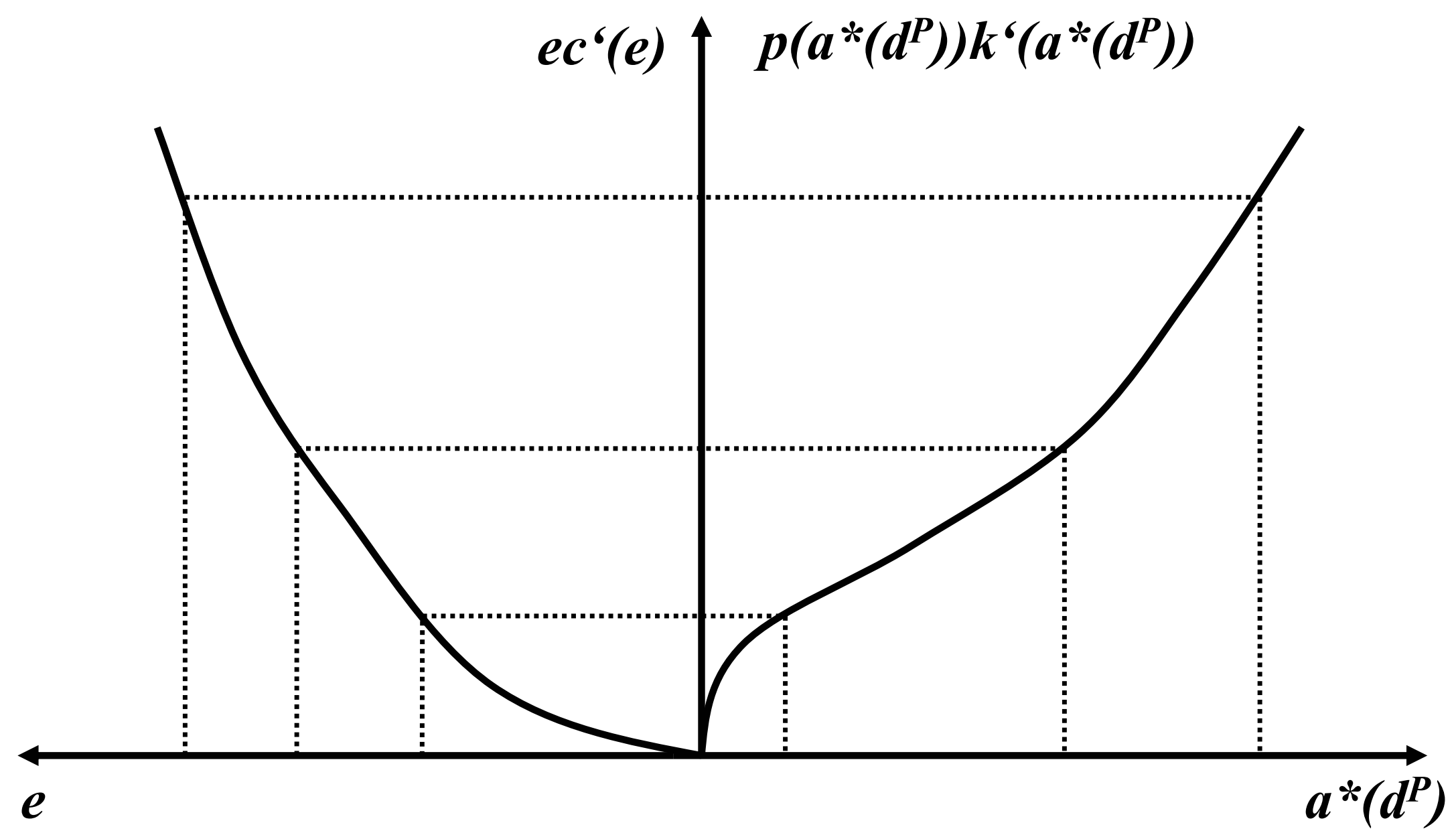

